\title{
Analytical ultracentrifugation: still the gold standard that offers multiple solutions
}

\author{
Renwick C. J. Dobson ${ }^{1,2} \cdot$ Trushar R. Patel $^{3}$
}

Published online: 19 November 2020

(c) European Biophysical Societies' Association 2020

\begin{abstract}
Understanding the nature of macromolecules and their interactions in solution underpins many fields, including biology, chemistry and materials science. The 24th International Analytical Ultracentrifugation Workshop and Symposium (AUC2019, held in Christchurch, New Zealand, August 2019), brought together 77 international delegates to highlight recent developments in the field. There was a focus on analytical ultracentrifugation, although we recognise that this is but one of the key methods in the biophysicist's toolkit. Many of the presentations showcased the versatility of analytical ultracentrifugation and how such experiments are integrated with other solution techniques, such as small-angle X-ray scattering, cryo-electron microscopy, isothermal titration calorimetry and more. This special issue emphasises a wide range of themes covered in the meeting, including carbohydrate chemistry, protein chemistry, polymer science, and macromolecular interactions.
\end{abstract}

\section{Introduction and conference organisation}

On a crisp spring morning in late August, Christchurch, New Zealand, gathered a 'rotor' (the collective noun) of biophysicists with a shared interest in the solution behaviour of biomolecules and polymers, and a focus on analytical ultracentrifugation (AUC). They had come together for AUC2019, the 24th International Analytical Ultracentrifugation Workshop and Symposium, to discuss and celebrate recent developments in the field.

As chair of the organising committee, Ren Dobson is very grateful to the 77 delegates who attended the event, most of whom were from Europe, North America, or Asia, and had made the long trip out to New Zealand (in some cases over

Renwick C. J. Dobson

renwick.dobson@canterbury.ac.nz

Trushar R. Patel

trushar.patel@uleth.ca

1 Biomolecular Interaction Centre and School of Biological Sciences, University of Canterbury, PO Box 4800, Christchurch 8140, New Zealand

2 Bio21 Molecular Science and Biotechnology Institute, Department of Biochemistry and Molecular Biology, University of Melbourne, Parkville, VIC 3010, Australia

3 Department of Chemistry and Biochemistry, Alberta RNA Research and Training Institute, University of Lethbridge, Lethbridge, AB T1K 3M4, Canada
2 days!). This was just the second time the meeting had been held in the southern hemisphere, but we hope not the last.

A special mention and thanks to the committees that helped shape AUC2019. The scientific organising committee had a strong international flavour, comprising Sarah Atkinson (Monash University, Australia), Olwyn Byron (University of Glasgow, UK), Helmut Cölfen (Universität Konstanz, Germany), Luitgard Nagel-Steger (Heinrich Heine Universität, Germany), Phillip Koldewey (F. Hoffmann-La Roche Ltd, Basel, Switzerland), Alexandra Solovyova (Newcastle University, UK), Borries Demeler and Trushar Patel (University of Lethbridge, Canada), Karen Fleming (Johns Hopkins University, US), Walter Stafford (Harvard Medical School, US), Susumu Uchiyama (Osaka University, Japan) and Ren Dobson (University of Canterbury, New Zealand). The local organising committee comprised: Ren Dobson, David Goldstone (University of Auckland, New Zealand), Michael Griffin (University of Melbourne, Australia), Chris Horne (University of Canterbury, New Zealand), Grant Pearce (University of Canterbury, New Zealand), and Tatiana Soares da Costa (La Trobe University, Australia).

Following the format of previous events, AUC2019 started with 3 days of workshops. There has always been a strong emphasis on training the next generation and this edition featured 21 (!) workshops from the experts in the field. Many of the workshops focused on data analysis using the various computational tools (UltraScan, SedFit, SedAnal, SedNterp, Sverdberg, Gussi and more) but there were also 
workshops on complementary techniques such as isothermal titration calorimetry, microscale thermophoresis, smallangle X-ray scattering and fluorescence spectroscopy. The workshops highlight not only the wealth of knowledge in the field, but also the keen willingness of the experts to share this knowledge and grow this branch of biophysics.

The symposium was opened with the Svedberg lecture, which is a prestigious honour that recognises significant achievements within the field. For AUC2019, the award went to Borries Demeler (University of Lethbridge, Canada), for his longstanding contributions to method development and analysis software. There was continued interest and discussion on the new Beckman Optima AUC. Although it was released several years ago, only recently has it gone beyond testing with a growing number of laboratories having now purchased the instrument. The concurrent development of analysis software to match the capabilities of the new instrument, particularly multiwavelength data collection, has caught up and we can expect a growing number of papers reporting these types of experiments.

The symposium provided a platform for students and emerging researchers to present their research-44\% of the oral presentations were from this group (Table 1). However, one area where the community (and the organisers of subsequent events) can improve is supporting the participation of women in the field. Just $26 \%$ of delegates were female-if we wish to grow the community, particularly in the context of fresh ideas and directions, then encouraging a broader church is the way to go.

\section{Outline of articles covered in 24th international analytical ultracentrifuge conference special volume}

This AUC2019 Conference Proceedings volume contains many exciting research and insightful methods of development articles. It covers a wide range of topics that can be loosely grouped in the following theme: general methods,

Table 1 Metrics for gender and career stage participation

\begin{tabular}{|c|c|c|c|c|c|}
\hline & \multicolumn{2}{|l|}{ Gender } & \multicolumn{3}{|c|}{ Career stage } \\
\hline & Female & Male & Student & Emerging & Established \\
\hline $\begin{array}{l}\text { Registra- } \\
\text { tions }\end{array}$ & $26 \%(19)$ & $74 \%(54)$ & & & \\
\hline $\begin{array}{r}\text { Session } \\
\text { chairs }\end{array}$ & $38 \%(6)$ & $62 \%(10)$ & 0 & 3 & 13 \\
\hline Talks & $31 \%(13)$ & $69 \%(28)$ & 5 & 13 & 23 \\
\hline Full talks & $42 \%(8)$ & $58 \%(11)$ & 1 & 5 & 13 \\
\hline Short talk & $20 \%(5)$ & $80 \%$ (17) & 4 & 8 & 10 \\
\hline
\end{tabular}

protein chemistry, polysaccharides, and nucleic acids and their interactions.

\section{General methods}

The fluorescence-detection system with AUC (AUC-FDS) offers many advantages over the absorbance and interference optics. For example, Crowther and colleagues employ the AUC-FDS to determine the hydrodynamic properties of $\beta$-lactoglobulin under its native condition-in milk (Crowther et al. (2020)). They also report the interactions of $\beta$-lactoglobulin with other proteins and suggest that immunoglobulins are likely their interacting partners. Since $\beta$-lactoglobulin is one of the most abundant components of cow and goat milk, information on their solution properties and interactions are of high significance. Another example of AUC-FDS is in the study of monoclonal antibodies (mAbs) under high non-ideal conditions reported by Correia et al. (Correia et al. 2020). Their analysis of simulated data provides insights into the improvements in the SEDNAL data analysis package. They also demonstrate that direct boundary global fitting that accounts for (1), the hydrodynamic parameter $k \mathrm{~s}$, (2), the thermodynamic parameter BM1, and (3), a weak association, provide a meaningful interpretation of data involving high-concentration systems, such as mAbs that are typically used at $\sim 50-200 \mathrm{mg} / \mathrm{mL}$ !

The Demeler laboratory developed novel calibration disks to facilitate accurate and reproducible calibration of Beckman Optima rotor stretch profiles (Stoutjesdyk et al. 2020a). Their calibration disks offer several advantages, including the mechanical rigidity at high rotor speed and accurate prediction of cell bottom boundary that in turn aids reliable fitting of the Lamm equation models. Also, the Demeler laboratory utilised the new Beckman Optima AUC and have developed a novel method to determine the compressibility of solvents. They validate their results by studying the compressibility of water and toluene. This approach is useful to predict boundary conditions for multispeed AUC experiments, which can be applied to study heterogeneous systems (Stoutjesdyk et al. 2020b).

Traditionally, only quartz windows have been utilised for AUC data collection using absorbance optics below $235 \mathrm{~nm}$, as the sapphire windows are unable to provide high-quality data collection at such wavelengths. Bhattacharya and von Seggern demonstrated that the new generation of sapphire windows provides improved data collection as compared to the current generation (Bhattacharya and Seggern 2020). Moreover, their work also suggests that new-generation sapphire windows provide results similar to quartz windows in absorbance mode at lower wavelengths, implying that they can also be used for absorbance data collection in the future.

The SV-AUC (sedimentation velocity-AUC) approach has been widely used to study purity, aggregation, and 
conformational changes of biomolecules. Brautigam and colleagues compared the $\mathrm{c}(\mathrm{s})$ distribution method with the difference sedimentation velocity (DSV) method to investigate the detection of ligand-induced conformational changes in proteins (Brautigam et al. (2020)). They also developed a new programme (DiSECT) to automate DSV data analysis and compared the benefits as well as challenges of each method.

\section{Protein chemistry}

The Peukert laboratory utilise multiwavelength analytical ultracentrifugation (MW-AUC) to investigate the effect of the length and diameter of amyloid fibrils on sedimentation coefficient distribution. They also demonstrate the fibril sizereduction kinetics using AUC. Amyloid fibrils are implicated in various fields including health and medicine, food and material science, as well as nanotechnology. Therefore, their detailed characterisation using AUC and other biophysical methods is of high significance (Uttinger et al. 2020). The next article from the Dobson laboratory demonstrated that the lid domain, which is one of the three key domains of bacterial pyruvate kinase enzymes, is not required for the catalytic activity of pyruvate kinase. Using AUC and SAXS, they demonstrated that a recombinant pyruvate kinase protein, lacking the lid domain is structurally similar to that of full-length wild-type pyruvate kinase. The biochemical analysis suggested that the recombinant enzyme devoid of the lid domain retains a low level of catalytical activity (Sugrue et al. 2020). The Comoletti laboratory designed a heterodimeric construct of Reelin protein, which is essential for neocortex development and synaptic function. They expressed, purified, and investigated the activity of recombinant heterodimeric Reelin. The protocol established by the authors allows the preparation of a high amount of recombinant protein, which can be further characterised using AUC to study its interactions with other binding partners (Turk et al. 2020).

\section{Polysaccharides}

AUC can also be utilised to characterise the solution properties of polysaccharides and their complexes. The Harding laboratory first demonstrated the application of sedimentation equilibrium-AUC (SE-AUC) to investigate the molar mass distribution of tert-butyldimethylsilyl chitosan derivative. Since the modified chitosan is only soluble in ethyl acetate and toluene, the authors developed a unique experimental strategy that is explained here (Wakefield et al. 2020a). In their second article, the Harding laboratory formulated and characterised the self-associative behaviour of two unique aminocelluloses using SV-AUC and SE-AUC methods. Their assessment of these aminocelluloses indicates that self-associative behaviour could be a useful property to strengthen archaeological wood samples (Wakefield et al. 2020b). As a long-term goal of the Harding laboratory is to develop polysaccharide-based material to restore the Oseberg ship, such experimental strategies involving AUC and other biophysical methods to characterise modified biopolymers are of high significance. Using the SV-AUC study, the Fisk laboratory suggested that aroma compounds such as aldehydes, ketones, and phenols interact with submaxillary mucin (Dinu et al. 2020). As the aroma compounds are being used in many industries, their work provides a framework for studying aroma compounds-mucin interactions, which can be further exploited using AUC and other biophysical methods.

\section{Nucleic acids and their interactions}

Compared to many other biophysical methods, SV-AUC offers the advantage of analysing hydrodynamic properties and homogeneity in solution, without involving any matrix (e.g. packing material for size-exclusion chromatography). The emergence of critical roles of non-coding RNAs has ignited huge interest in their structural studies. However, as RNA molecules are flexible and require careful optimisation of buffer components for structural studies, their purity must be assessed by a reliable method. Therefore, the Patel laboratory contribute an article that demonstrates the application and benefits of SV-AUC to evaluate solution properties and homogeneity of non-coding RNAs (Badmalia et al. 2020).

At the heart of gene regulation are the interactions between nucleic acids and the proteins that act to repress or promote transcription. In some cases, the stoichiometry of this interaction is not simple. The Dobson lab provides a good example where the sedimentation velocity method with multiwavelength data collection is perfectly suited for answering the question of stoichiometry. In their system, it is clear that multiple protein species bind the DNA operator sequence. They take advantage of the good orthogonality between the spectra for protein and DNA, making deconvolution of the data possible. With these data in hand, they demonstrate that multiple dimers of the NanR repressor bind to the operator sequence (Horne et al. 2020).

\section{Conclusions}

In conclusion, the 24th International Analytical Ultracentrifugation Workshop and Symposium was a huge success. This event provided opportunities to students, postdoctoral fellows, faculty members, and industry representatives to network, share their ideas, obtain constructive feedback, and to deliver a strong message that AUC continues to be a 
critical tool for quality control and to investigate a range of molecules and their complexes.

Finally, we look forward to the next edition of this longrunning event, which will be held in Lethbridge, Canada and will be organised by Borries Demeler and Trushar Patel.

Acknowledgments We acknowledge the sponsors that made AUC2019 possible. We are especially grateful to our major sponsor, Beckman Coulter Life Sciences, who have sponsored the event over many years. We thank the following for financial support: Izon, Roche, and the Biomolecular Interaction Centre (University of Canterbury). In addition, we thank the following for providing with travel support to delegates (largely students): the Maurice Wilkins Centre for molecular biodiscovery, the Riddet Institute and the Northwest Biophysics Consortium. Finally, we thank Rob Gilbert for supporting the publication of this special issue.

\section{References}

Badmalia MD, Siddiqui MQ, Mrozowich T et al (2020) Analytical ultracentrifuge: an ideal tool for characterization of non-coding RNAs. Eur Biophys J. https://doi.org/10.1007/s00249-020-01470 $-9$

Bhattacharya A, Von Seggern E (2020) A comparison of data quality using quartz vs. sapphire cell windows in analytical ultracentrifugation. Eur Biophys J. https://doi.org/10.1007/s00249-020-01454 $-9$

Brautigam CA, Tso SC, Deka RK et al (2020) Using modern approaches to sedimentation velocity to detect conformational changes in proteins. Eur Biophys J. https://doi.org/10.1007/s0024 9-020-01453-w

Correia JJ, Wright RT, Sherwood PJ et al (2020) Analysis of nonideality: insights from high concentration simulations of sedimentation velocity data. Eur Biophys J. https://doi.org/10.1007/s0024 9-020-01474-5

Crowther JM, Broadhurst M, Laue TM et al (2020) On the utility of fluorescence-detection analytical ultracentrifugation in probing biomolecular interactions in complex solutions: a case study in milk. Eur Biophys J. https://doi.org/10.1007/s00249-020-01468-3
Dinu V, MacCalman T, Yang N, Adams GG, Yakubov GE, Harding SE, Fisk ID (2020) Probing the effect of aroma compounds on the hydrodynamic properties of mucin glycoproteins. Eur Biophys J. https://doi.org/10.1007/s00249-020-01475-4

Horne CR, Henrickson A, Demeler B, Dobson RCJ (2020) Multiwavelength analytical ultracentrifugation as a tool to characterise protein-DNA interactions in solution. Eur Biophys J. https://doi. org/10.1007/s00249-020-01481-6

Stoutjesdyk M, Henrickson A, Minors G et al (2020) A calibration disk for the correction of radial errors from chromatic aberration and rotor stretch in the Optima AUC ${ }^{\mathrm{TM}}$ analytical ultracentrifuge. Eur Biophys J. https://doi.org/10.1007/s00249-020-01434-z

Stoutjesdyk M, Brookes E, Henrickson A et al (2020) Measuring compressibility in the Optima AUC ${ }^{\mathrm{TM}}$ analytical ultracentrifuge. Eur Biophys J. https://doi.org/10.1007/s00249-020-01482-5

Sugrue E, Coombes D, Wood D et al (2020) The lid domain is important, but not essential, for catalysis of Escherichia coli pyruvate kinase. Eur Biophys J. https://doi.org/10.1007/s00249-020-01466 $-5$

Turk LS, Mitchell D, Comoletti D (2020) Purification of a heterodimeric Reelin construct to investigate binding stoichiometry. Eur Biophys J. https://doi.org/10.1007/s00249-020-01465-6

Uttinger MJ, Heyn TR, Jandt U et al (2020) Measurement of length distribution of beta-lactoglobulin fibrils by multiwavelength analytical ultracentrifugation. Eur Biophys J. https://doi.org/10.1007/ s00249-020-01421-4

Wakefield JMK, Braovac S, Kutzke H et al (2020) Tert-butyldimethylsilyl chitosan synthesis and characterization by analytical ultracentrifugation, for archaeological wood conservation. Eur Biophys J. https://doi.org/10.1007/s00249-020-01450-z

Wakefield JMK, Hampe R, Gillis RB et al (2020) Aminoethyl substitution enhances the self-assembly properties of an aminocellulose as a potential archaeological wood consolidant. Eur Biophys J. https ://doi.org/10.1007/s00249-020-01451-y

Publisher's Note Springer Nature remains neutral with regard to jurisdictional claims in published maps and institutional affiliations. 